\title{
Dynamic Modeling and Ground Test of Tethered-net
}

\author{
Chen Qing-quan, Zhang Qing-bin, Tang Qian-gang \\ College of Aeronautics and Astronautics, National University of Defense Technology, Changsha 410073, Hunan, China
}

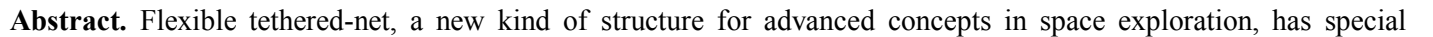

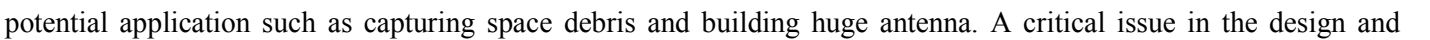

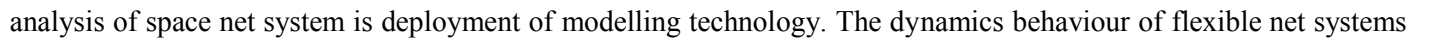

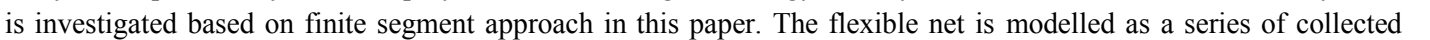

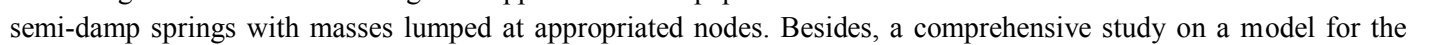

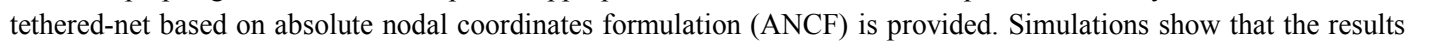

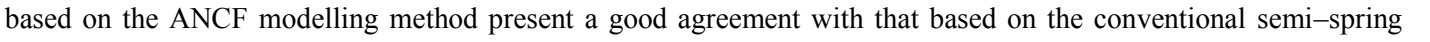

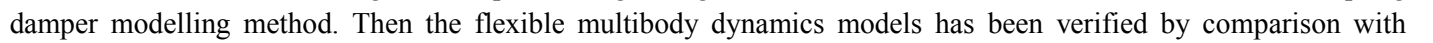

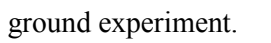

\section{Introduction}

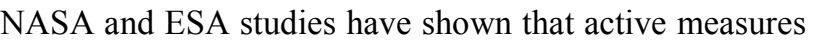

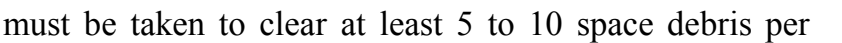

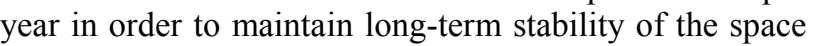

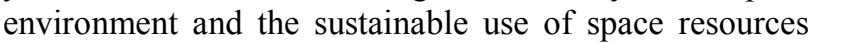

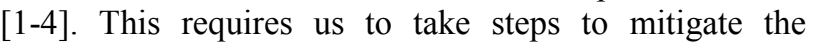

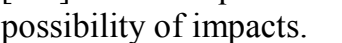

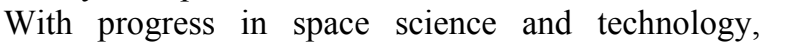

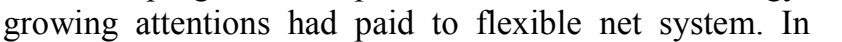

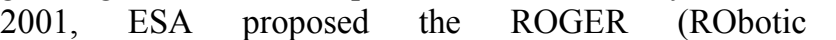

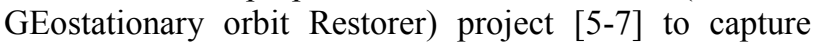

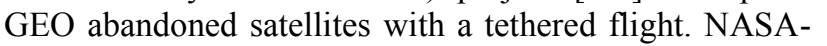

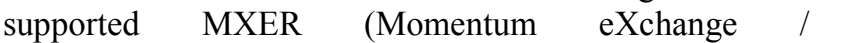

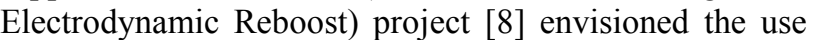

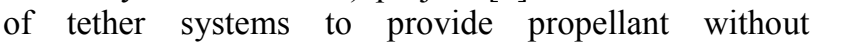

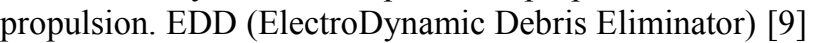

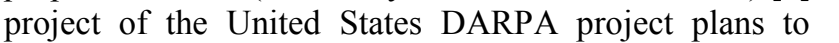

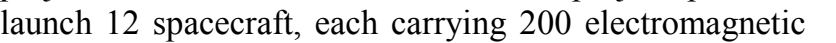

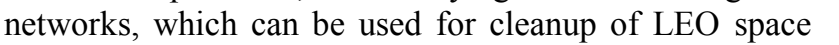

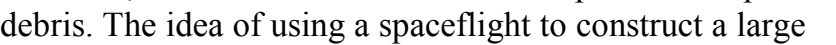

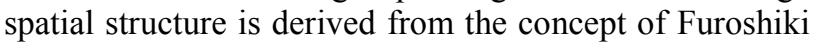

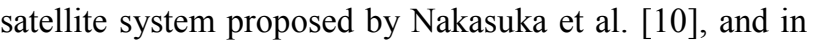

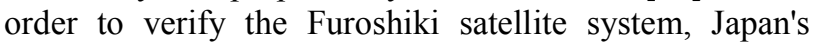

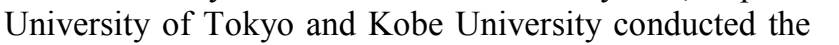
ए

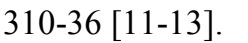

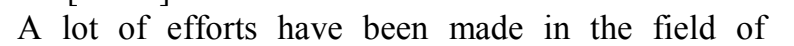

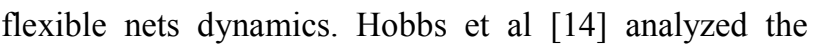
ए

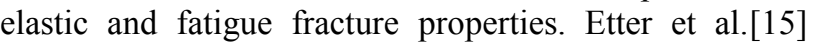

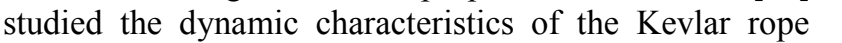
पा

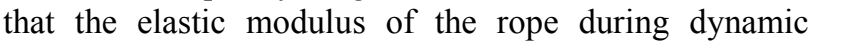

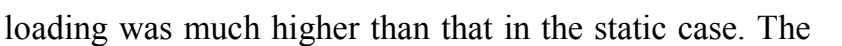

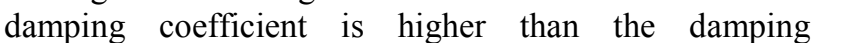

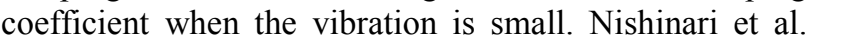

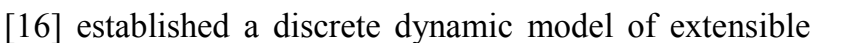

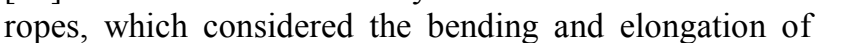
एवा山ाणाए

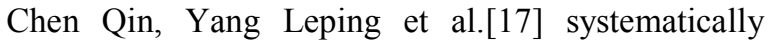

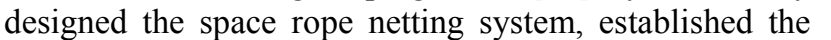

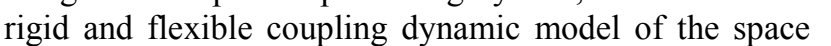

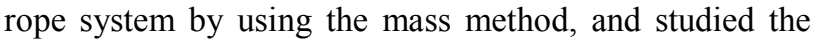

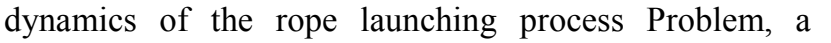

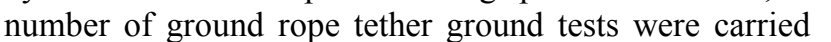

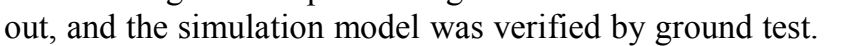

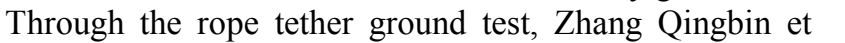

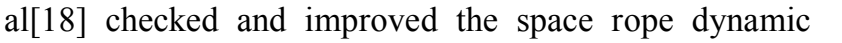

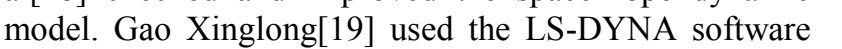

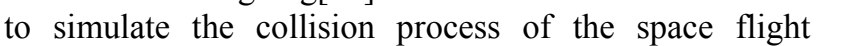

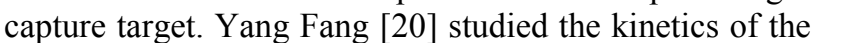
एा।

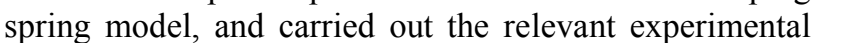

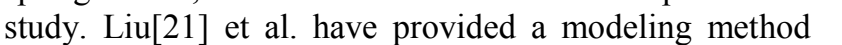

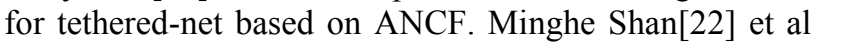

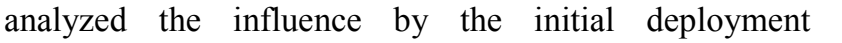

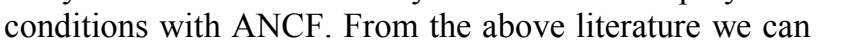

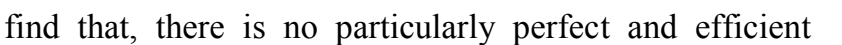

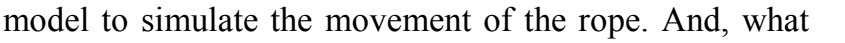

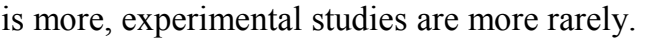

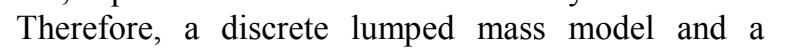

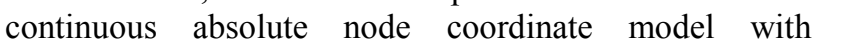

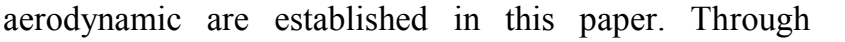

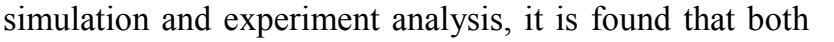

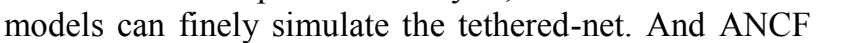

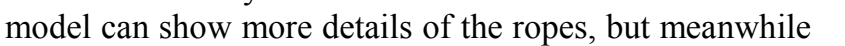

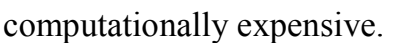




\section{ANCF model}

In ANCF, which is initially proposed by Shabana and utilized in solving large displacement and deformation problems, absolute $\square \square \square \square$ and the gradients of the positions act as the element nodal coordinates to describe

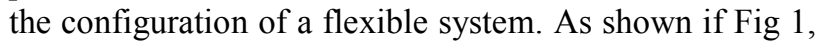
the nodal coordinates of the elements are defined in a global inertial coordinate frame $\left(O \boldsymbol{e}_{\square}^{(!)} \boldsymbol{e}_{\square}^{(!)} \boldsymbol{e}_{\square}^{(!)}\right) \square$

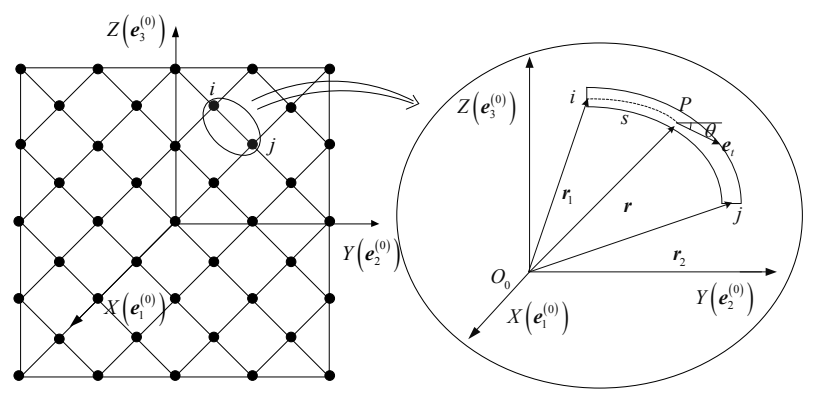

Figure 1. $\square \square \square \square \square \square \square \| ा \square$

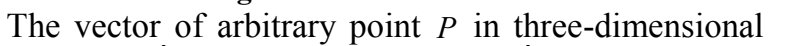

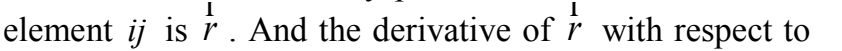

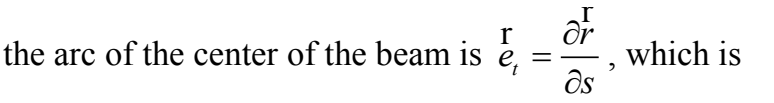

$$
\begin{aligned}
& r^{())}=\left(\begin{array}{lll}
X & Y & Z
\end{array}\right)^{T} \\
& e_{t}^{(\sqsupset)}=\left(\begin{array}{lll}
\partial X / \partial s & \partial Y / \partial s & \partial Z / \partial s
\end{array}\right)^{T}
\end{aligned}
$$

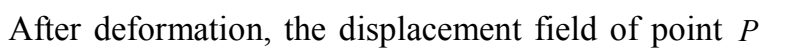

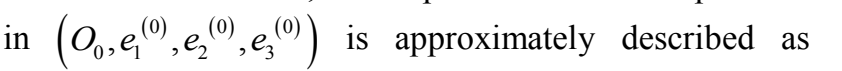

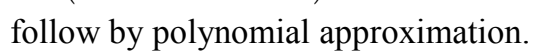

$$
\left.\begin{array}{l}
X=a_{\square}+a_{\square} s+a_{\square} s^{\square}+a_{\square} s^{\square} \\
Y=a_{\square}+a_{\square} s+a_{\square} s^{\square}+a_{\square} s^{\square} \\
Z=a_{\square}+a_{\square} s+a_{\square} s^{\square}+a_{\square} s^{\square} \\
\partial X / \partial s=a_{\square}+\square a_{\square} s+\square a_{\square} s^{\square} \\
\partial Y / \partial s=a_{\square}+\square a_{\square} s+\square a_{\square} s^{\square} \\
\partial Z / \partial s=a_{\square}+\square a_{\square} s+\square a_{\square} s^{\square}
\end{array}\right\}
$$

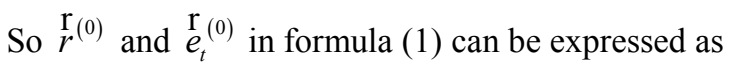

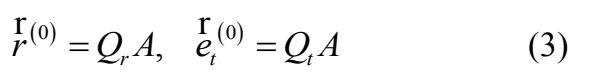

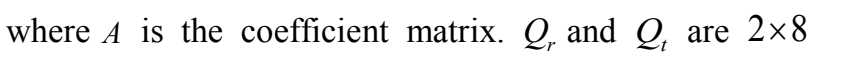

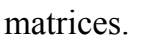

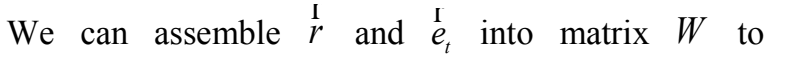

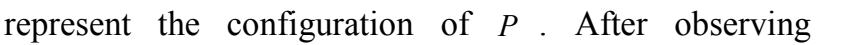

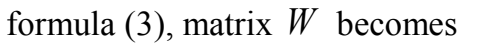

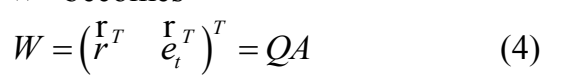

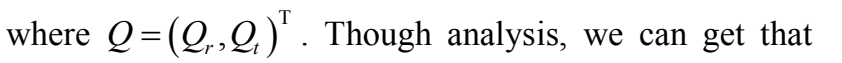

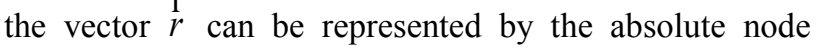

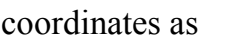

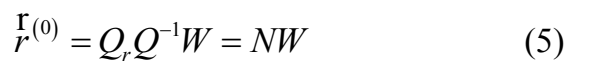

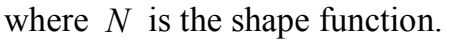

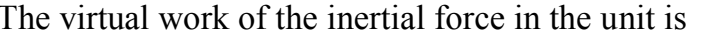

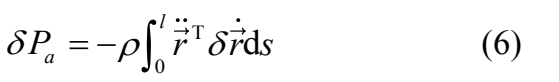

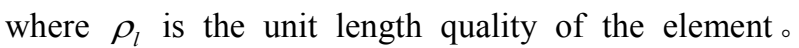

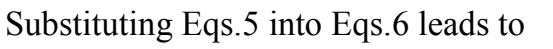

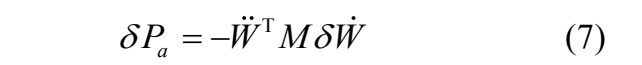

$\square \square \mid \Pi \square M=\rho \int_{\square}^{l} N \square N \square$ 。

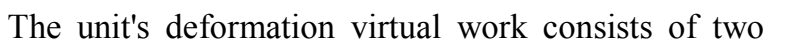

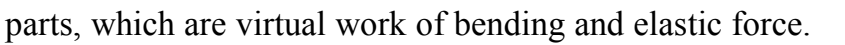
$\square \square[\|ा\| ा \|$

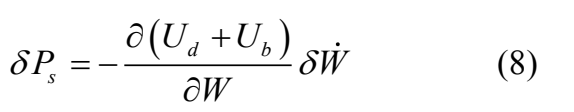

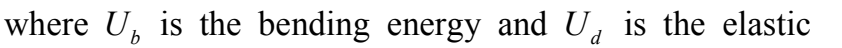

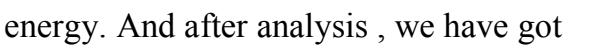

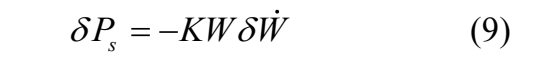

$\square \square \square K=\int_{\square}^{l} E A\left[\square W^{\square}\left(N_{s}^{\square} N_{s}\right) W^{\square}-N_{s}^{\square} N_{s}\right] \square s \square$

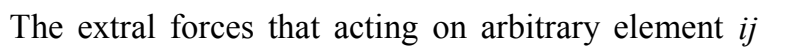

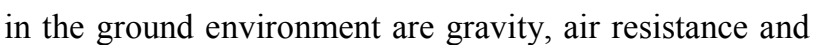

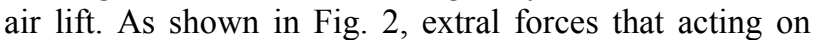

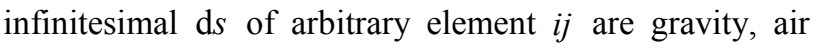

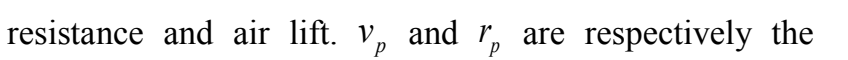

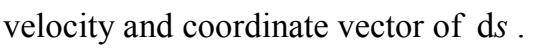

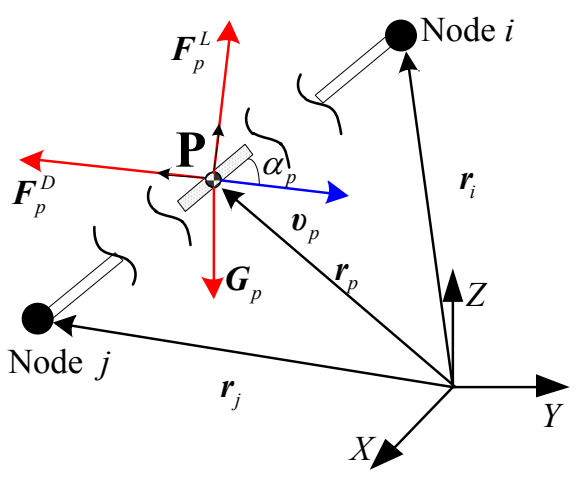

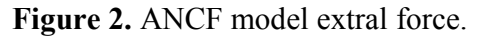

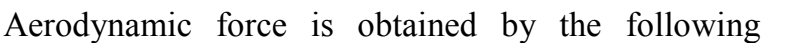
एणापण

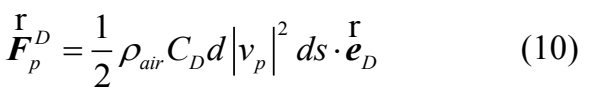

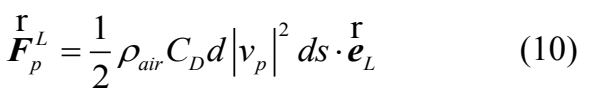

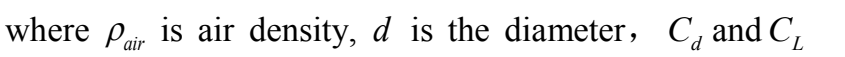

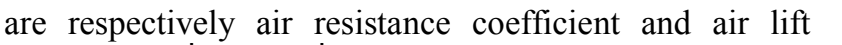

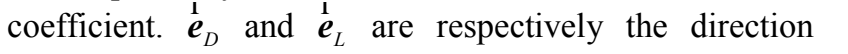
पाm

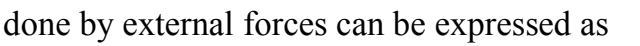




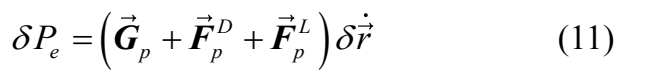

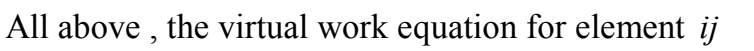
पाणाणा

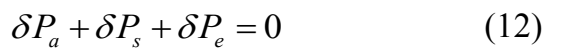

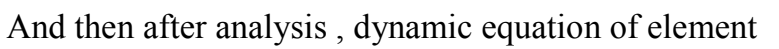
$i j$ 血口

$$
-\ddot{W}^{\square} M-K W+\boldsymbol{N}^{\square}\left(\boldsymbol{G}_{p}+\boldsymbol{F}_{p}^{D}+\boldsymbol{F}_{p}^{L}\right)=\square \square \square \square \square
$$

\section{Semi-spring damper model}

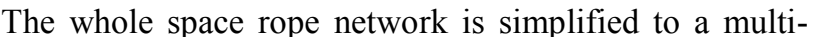

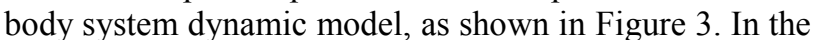

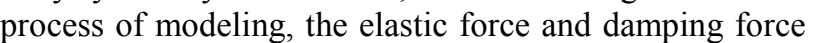

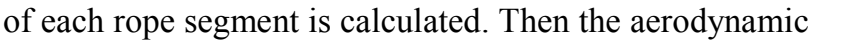

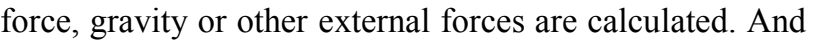

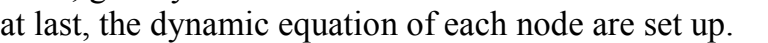
$\square$

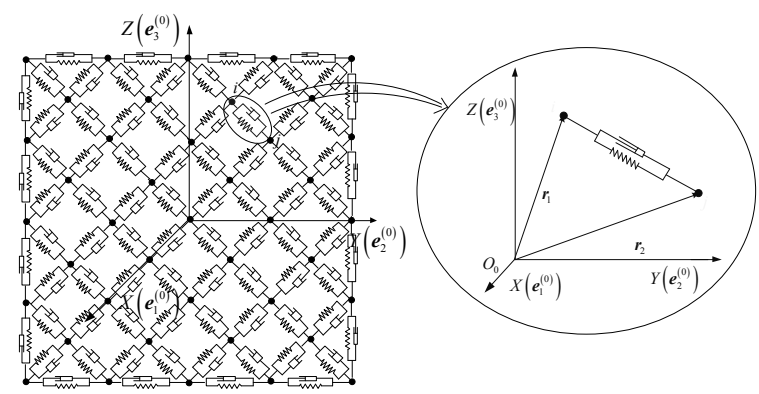

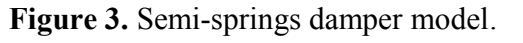

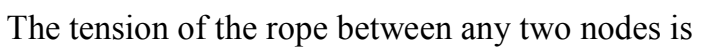

$$
T_{i j}=\left\{\begin{array}{cl}
f\left(l_{i j}\right) & l_{i j}>l_{i j}^{\square} \\
\square & l_{i j} \leq l_{i j}^{\square}
\end{array}\right.
$$

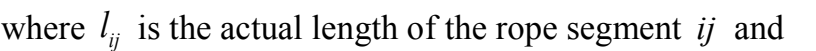

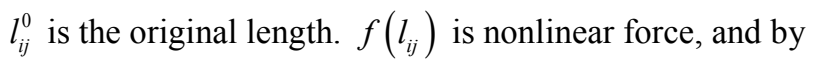

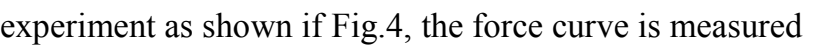

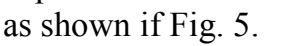
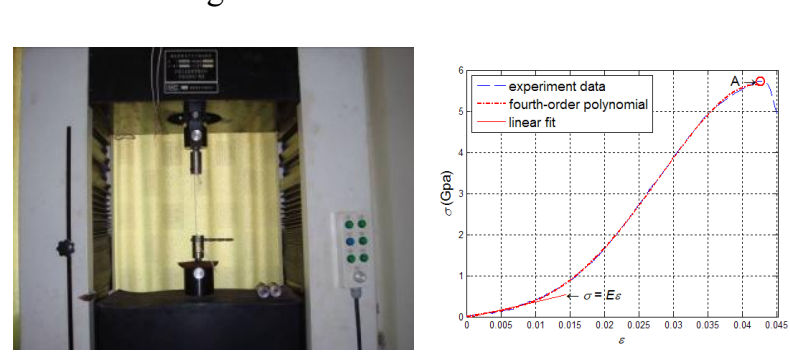

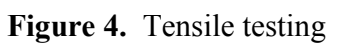

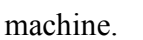

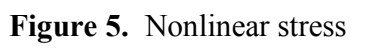

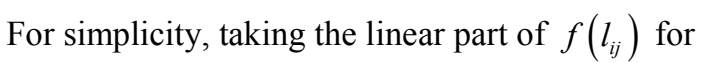

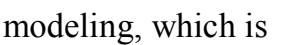

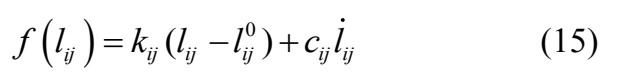

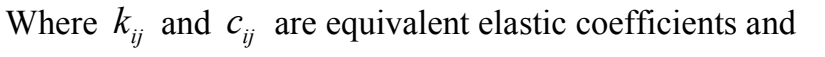

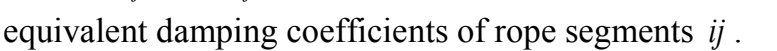

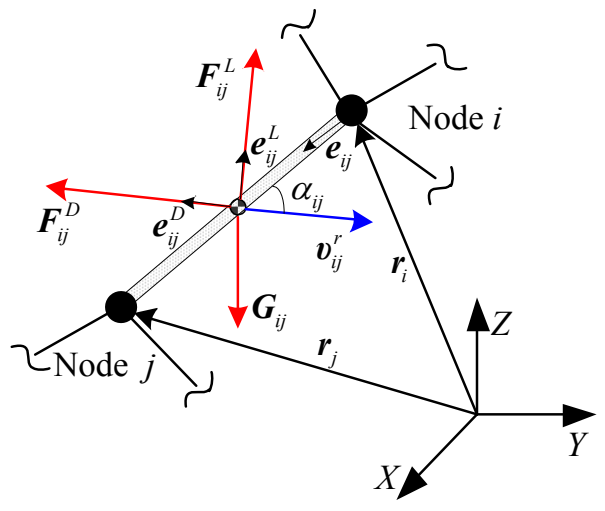

Figure 6.

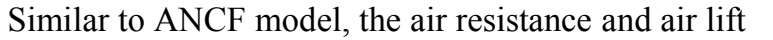
पाII

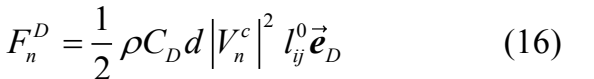

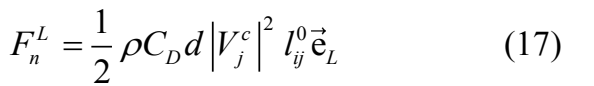

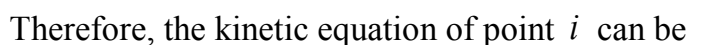
पाणाणाणाए

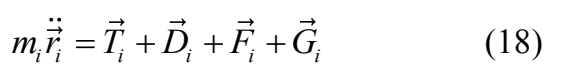

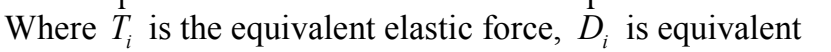

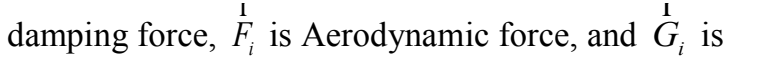
पणाणाणा

$$
\begin{aligned}
& \vec{T}_{i}=\sum_{n \in R_{(i)}} \vec{T}_{n}
\end{aligned}
$$

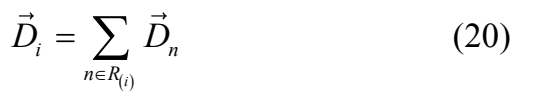

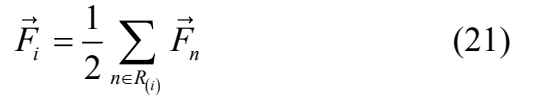

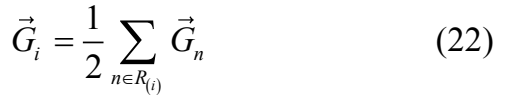

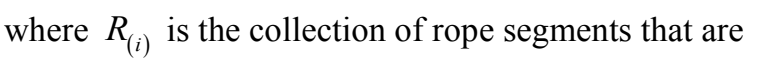

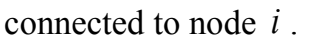

\section{Ground test}

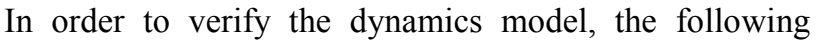

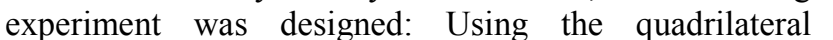

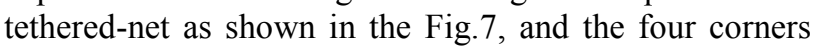

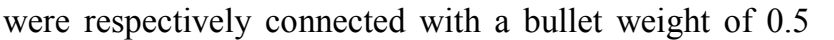

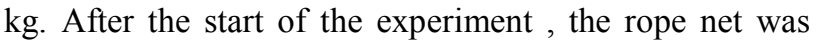

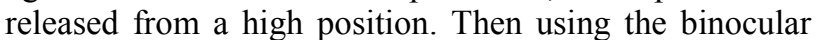

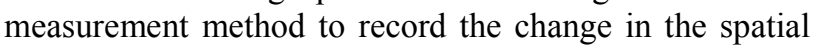

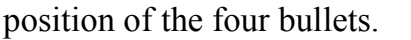




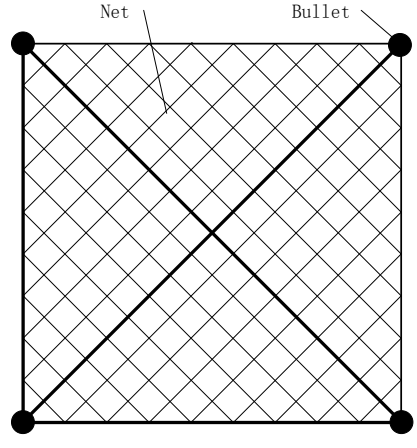

Figure 7.

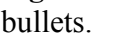

$\square$

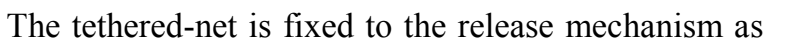

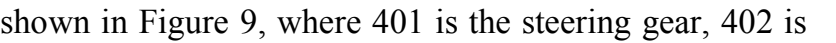

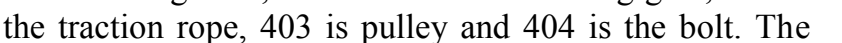

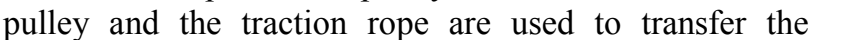

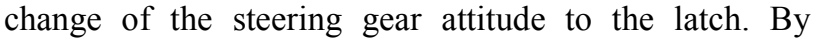

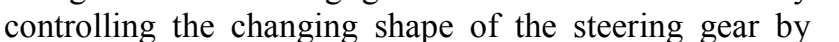

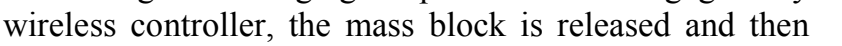

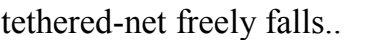
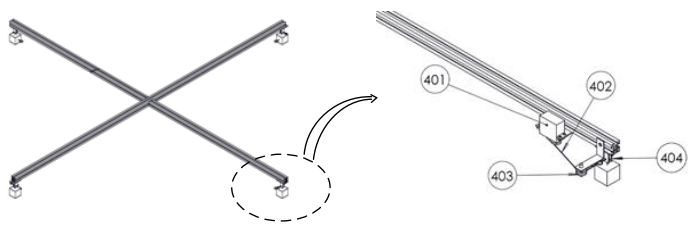

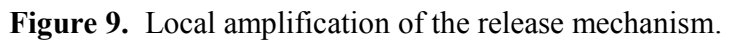

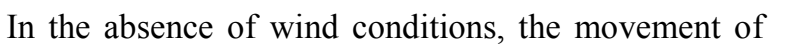

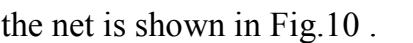

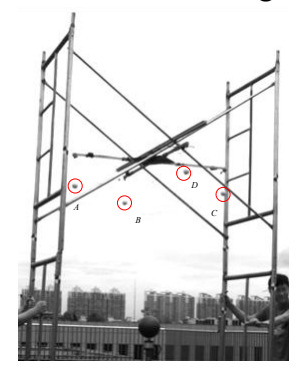

$\square \square \square$

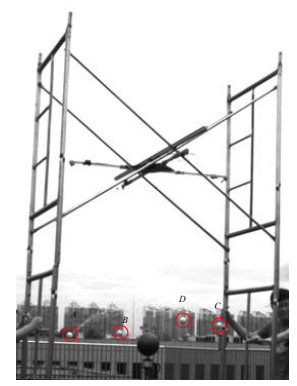

पाIIII

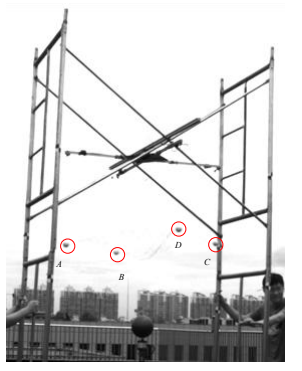

$\square[1 \square$

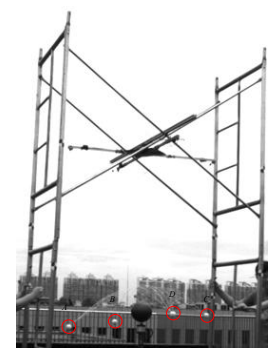

$\square[10$

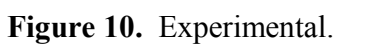

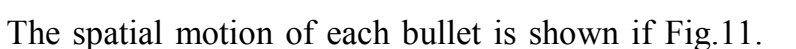

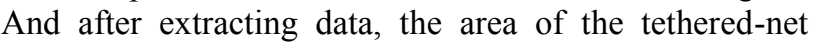

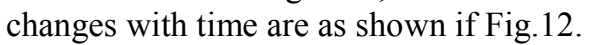

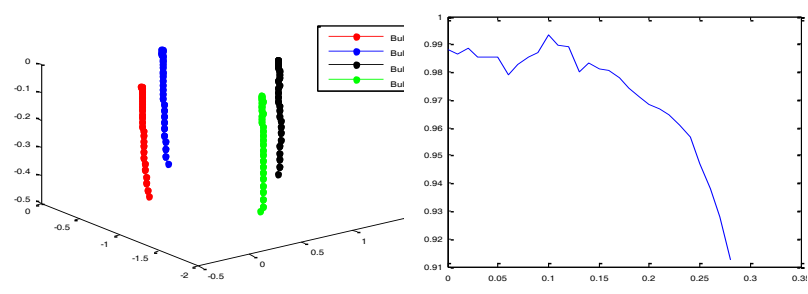

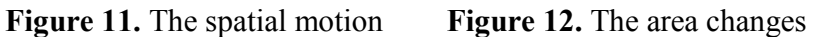

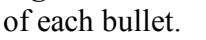

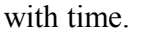

\section{Simulation and analysis}

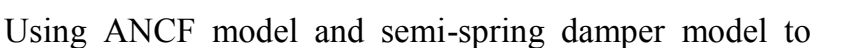

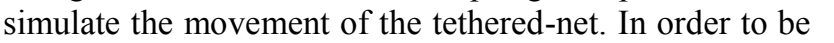

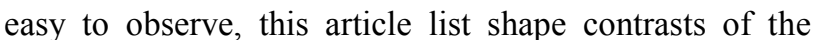

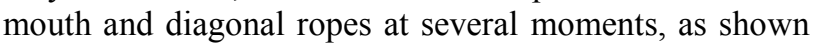

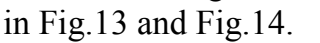
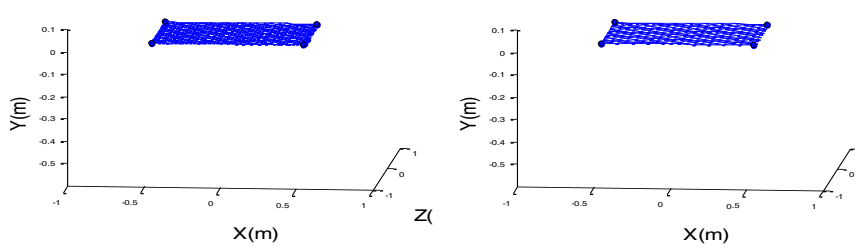

$\square \square$
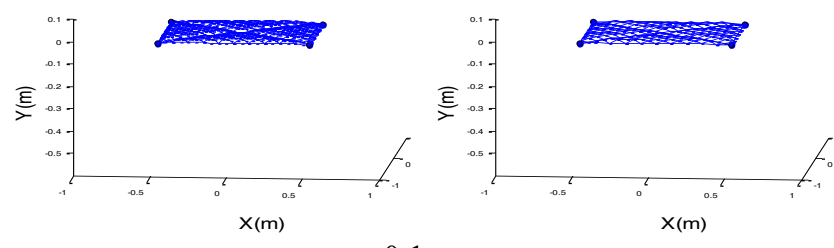

$\square[10$
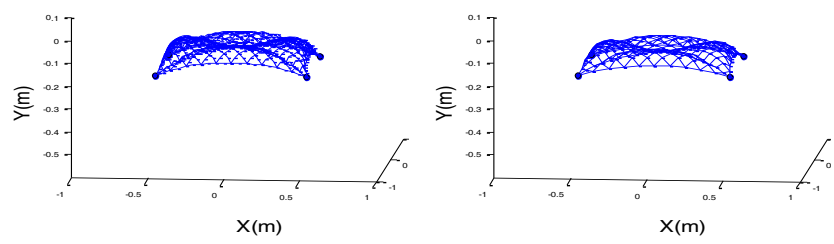

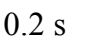

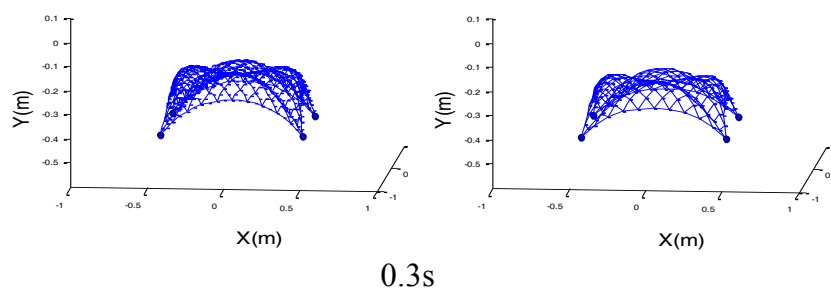

Figure 13. $\mathbb{\|} \square \square \square$

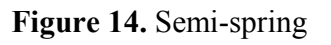
पापाणा

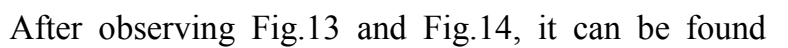

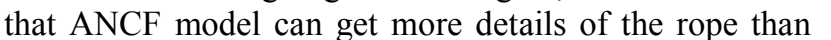

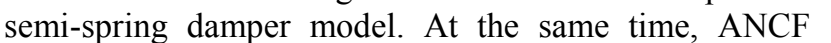

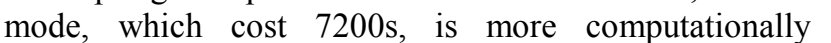

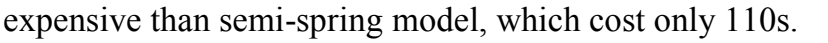

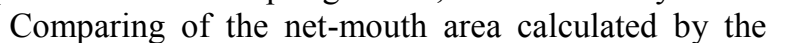

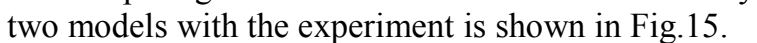




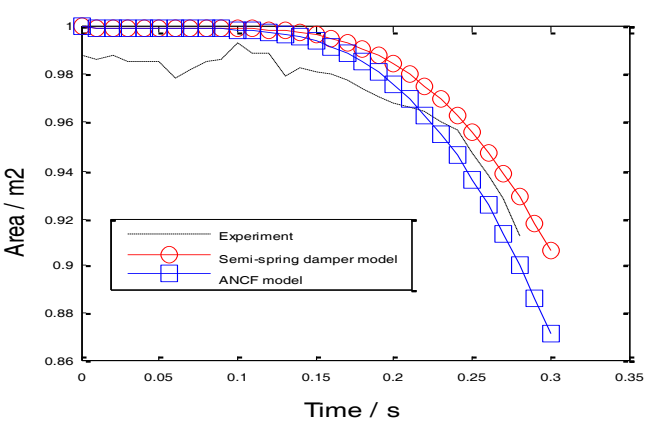

Figure 15.

$\square$

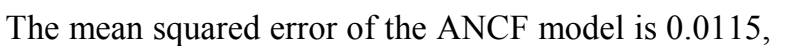
पा

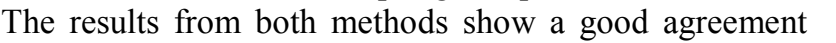

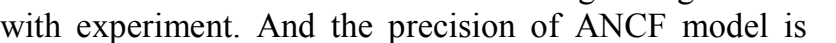
एणाएणा

\section{Conclusion}

$\square \square \square$ article established the aerodynamic Semi-spring damper model and ANCF model of the tethered-net system. And the $\square\|\| \| \square$ from both methods show a good agreement with experiment. The results indicate that the ANCF model is very suitable to describe the dynamics of a tethered-net. Furthermore, ANCF model is more capable of describing the flexibility of the net with fewer nodes than the conventional semi-spring damper model. However, it is more computationally expensive. Based on the comparison of single-step computational time, mass spring model is nearly 66 times faster than ANCF model.

\section{References}

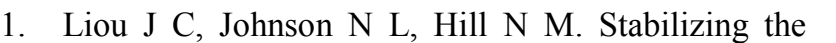

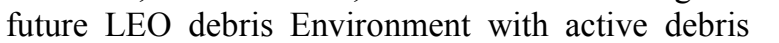

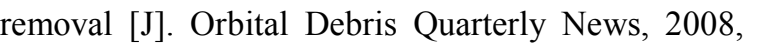
पाणाणाणा

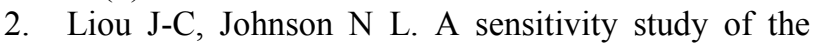

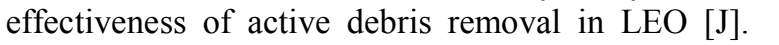

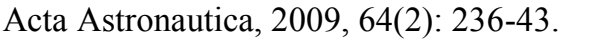

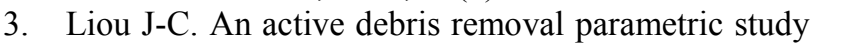

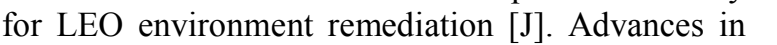

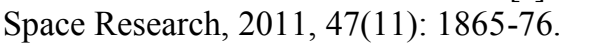

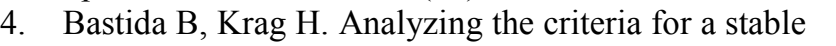

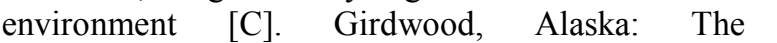

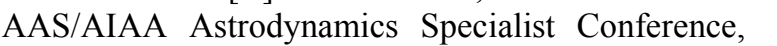
$\square 10 \mathrm{D}$

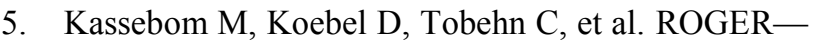

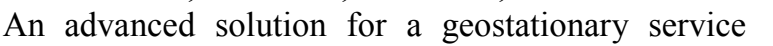

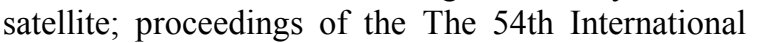

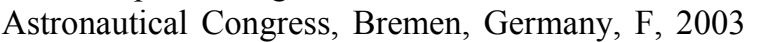

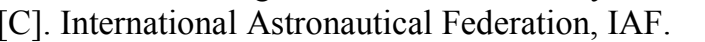

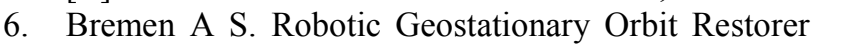

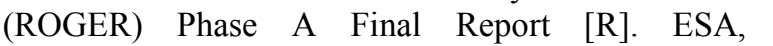

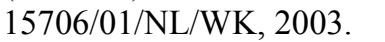

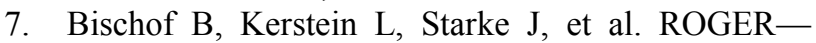

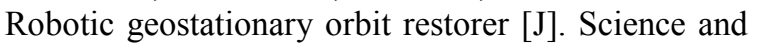

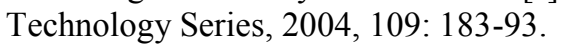

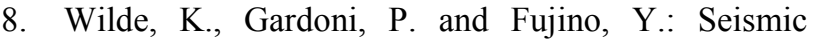

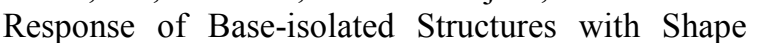

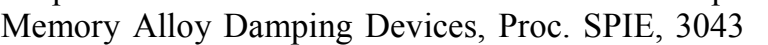

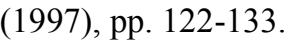

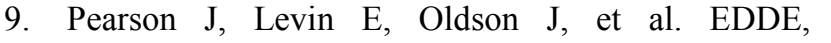

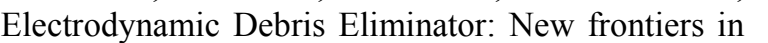

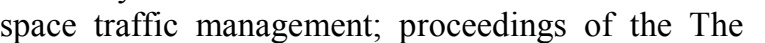

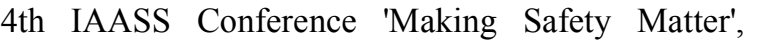

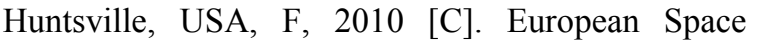
$\square \square \| 11 \square$

$\square \square$ Nakasuka S, Aoki T, Ikeda I, et al. "Furoshiki

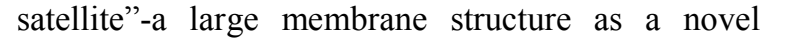

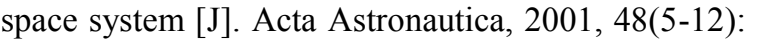

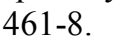

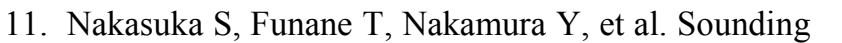

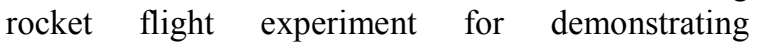
"Furoshiki Satellite" for large phased array antenna

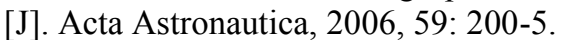

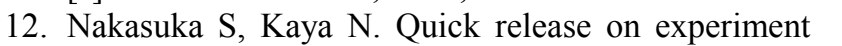

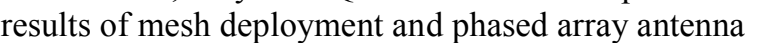

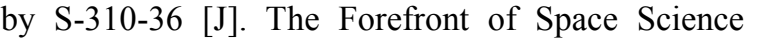

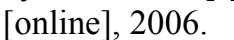

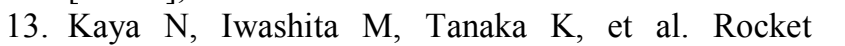

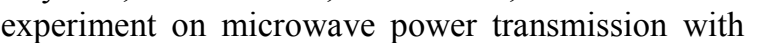

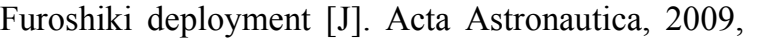

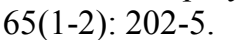

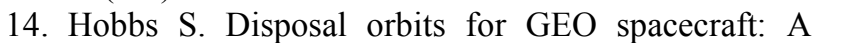

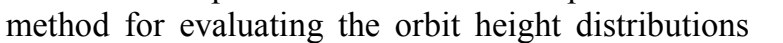

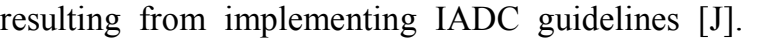

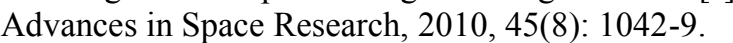

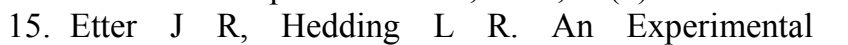

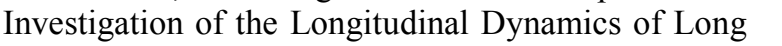

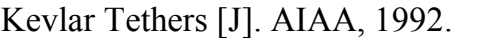

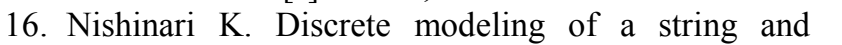

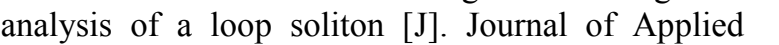

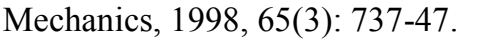

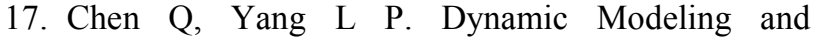

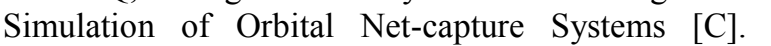

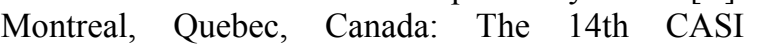

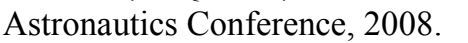

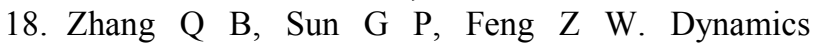

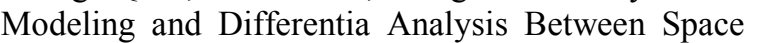

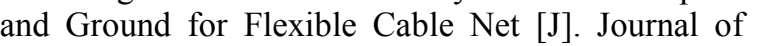

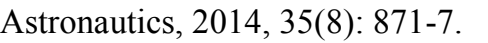

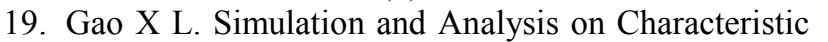

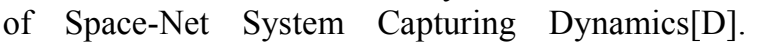

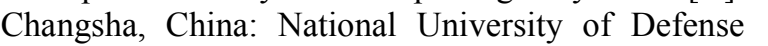

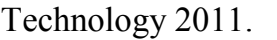

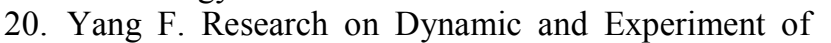

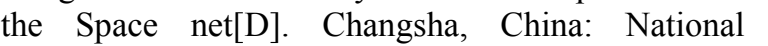

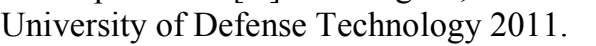

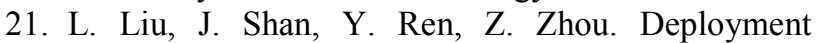

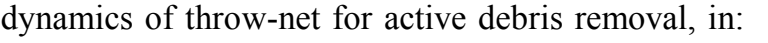

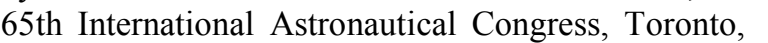

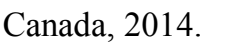

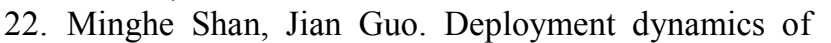

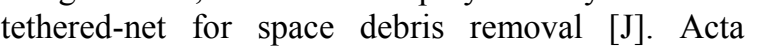

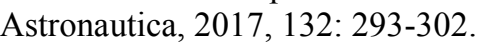

